Bundesgesundheitsbl 2020 $63: 796$ https://doi.org/10.1007/s00103-020-03135-6 Online publiziert: 21. April 2020

(c) Springer-Verlag GmbH Deutschland, ein Teil von Springer Nature 2020

\title{
Erratum zu: Stellungnahme S 19 Fehlanwendungen von Blutkomponenten
}

\section{Erratum zu:}

Bundesgesundheitsbl 2019

https://doi.org/10.1007/s00103-01902989-9

Die Originalveröffentlichung der Stellungnahme enthält eine Ungenauigkeit in Bezug auf die Meldeverpflichtung für „near misses“ („Beinahe-Schäden“; Seite 1141,3 . Spalte).

Nur wenn die Ursache einer Fehltransfusion in einer fehlerhaften Zuordnung bei der Herstellung oder dem Transport der Blutkomponente liegt, muss dies der Bundesoberbehörde gemeldet werden.

Der entsprechende Absatz lautet im Folgenden korrekt:

Wird eine Fehltransfusion bekannt, die für die Blutprodukte empfangende Person folgenlos, d.h. ohne transfusionsmedizinische Reaktion oder klinischen Schaden, abläuft, besteht dennoch für die Zulassungsinhaberin oder den Zulassungsinhaber die Verpflichtung, dies als schwerwiegenden Zwischenfall der Bundesoberbehörde zu melden, wenn die Ursache der Fehltransfusion in einer fehlerhaften Zuordnung bei der Herstellung oder dem Transport der Blutkomponenten liegt ( $\$ 63 \mathrm{i}$ Abs. $6 \mathrm{AMG})$. 